\title{
The development of a simple questionnaire to screen patients with SLE for the presence of neuropsychiatric symptoms in routine clinical practice
}

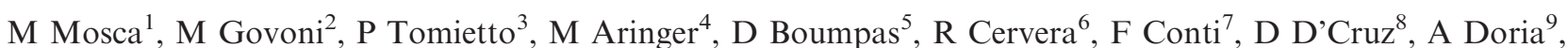
D De La Fuente ${ }^{10}$, M Galeazzi ${ }^{11}$, F Houssiau ${ }^{12}$, TWJ Huizinga ${ }^{13}$, MA Khamashta ${ }^{8}$, L Ines ${ }^{14}$, C Duarte ${ }^{14}$, M Couto ${ }^{14}$, P Meroni $^{15}$, C Montecucco ${ }^{16}$, E Norkuviene ${ }^{17}$, G Riemekasten ${ }^{18}$, V Rios ${ }^{10}$, M Schneider ${ }^{19}$, Y Shoenfeld ${ }^{20}$, GM Steup-Beekman ${ }^{13}$, M Szmyrka-Kaczmarek ${ }^{21}$, C Tani ${ }^{1}$, A Tincani ${ }^{22}$, AG Tzioufas ${ }^{23}$, R Voll ${ }^{24}$, W Bencivelli ${ }^{1}$, F Salaffi $^{25}$ and $\mathrm{S}$ Bombardieri ${ }^{1}$

${ }^{1}$ Rheumatology Unit. Department of Internal Medicine, University of Pisa, Italy; ${ }^{2}$ Section of Rheumatology, Department of Clinical and Experimental Medicine, University of Ferrara, Italy; ${ }^{3}$ Rheumatology Clinic, University of Udine, Udine, Italy; ${ }^{4}$ Division of Rheumatology, Department of Medicine III, University Clinical Center Carl Gustav Carus at the Technical University of Dresden, Germany; ${ }^{5}$ University of Crete Medical School, Heraklion, Crete, Greece; ${ }^{6}$ Department of Autoimmune Diseases, Hospital Clinic, Barcelona, Catalonia, Spain; ${ }^{7}$ Dipartimento di Clinica e Terapia Medica, Sezione di Reumatologia, Sapienza Università di Roma, Policlinico Umberto I, Roma, Italy; ${ }^{8}$ Lupus Research Unit, The Rayne Institute, St Thomas Hospital, London, UK; ${ }^{9}$ Division of Rheumatology, University of Padova, Padova, Italy; ${ }^{10}$ Department of Rheumatology, Hospital Universitari Bellvitge, Barcelona, Spain; ${ }^{11}$ Department of Clinical Medicine and Immunological Science, Rheumatology Unit, University of Siena, Siena, Italy; ${ }^{12}$ Rheumatology Department, Université Catholique de Louvain, Cliniques Universitaires Saint-Luc, Belgium; ${ }^{13}$ Department of Rheumatology, Leiden University Medical Centre, Leiden, The Netherlands; ${ }^{14}$ Rheumatology Department, University Hospital of Coimbra, Portugal; ${ }^{15}$ Department of Internal Medicine, Cattedra di Reumatologia, University of Milan, Istituto Ortopedico Gateano Pini, Milano, Italy; ${ }^{16}$ Cattedra di Reumatologia, IRCCS, Policlinico S. Matteo, Università di Pavia, Pavia, Italy; ${ }^{17}$ Rheumatology department, Kaunas Medical University Hospital, Lithuania; ${ }^{18}$ Department of Rheumatology and Clinical Immunology, Charité-Universitätsmedizin Berlin, Germany; ${ }^{19}$ Rheumatology, Clinic of Endocrinology, Diabetology and Rheumatology, Heinrich-Heine-University, Duesseldorf, Germany; ${ }^{20}$ Sheba Medical Center. Tel-Aviv University, Israel; ${ }^{21}$ Department of Rheumatology and Internal Medicine, Wroclaw University of Medicine, Poland;

${ }^{22}$ UO Reumatologia e Immunologia Clinica, Spedali Civili e Università degli Studi, Brescia, Italy; ${ }^{23}$ Department of Pathophysiology,

Medical School, National University of Athens, Greece; ${ }^{24}$ IZKF-N2 and Department of Internal Medicine 3 (Rheumatology, Immunology),

Nikolaus-Fiebiger Center, University of Erlangen-Nürnberg, Germany; and ${ }^{25}$ Cattedra di Reumatologia, Università, Politecnica delle Marche, Jesi, Ancona, Italy
\end{abstract}

\begin{abstract}
Aim: The creation of a physician-administered questionnaire to screen patients with Systemic Lupus Erythematosus (SLE) for the presence of symptoms suggestive of neuropsychiatric involvement (NPSLE).

Methods: The development of the questionnaire followed three phases. First, a list of manifestations was prepared based on the ACR case definitions for NPSLE. A first questionnaire was constructed including 119 items. To reduce their number, a Delphi analysis was carried out and a second questionnaire with 62 questions was developed. This questionnaire was administered to 139 patients with SLE (58 with NPSLE: 29 active, 29 inactive; and 81 without NPSLE: 39 active, 42 inactive). Questions relevant to the screening of patients were selected on the basis of the receiver operating characteristic (ROC) curve analysis.

Results: Twenty-seven questions concerning central nervous system and psychiatric manifestations were found to be relevant; the remaining could be eliminated without significantly affecting AUC. The area under the ROC curve (AUC) was 0.69 (95\% CI 0.61-0.78). A score above 17 was considered as suggestive of the presence of NPSLE with a sensitivity of $92.9 \%$ $(95 \%$ CI $85.1-97.3 \%)$ and specificity of $25.4 \%(95 \%$ CI $14.7-39.00 \%)$.

Conclusions: This questionnaire could represent a 'core set' of questions that could help in clinical practice to identify patients with neuropsychiatric symptoms requiring further evaluation. Lupus (2011) 20, 485-492.
\end{abstract}

Key words: Clinical practice; neuropsychiatric lupus; questionnaire; screening; systemic lupus erythematosus

Correspondence to: Marta Mosca, MD, Rheumatology Unit, Department of Internal Medicine, University of Pisa, Via Roma 67, 56126 Pisa, Italy

Email: marta.mosca@int.med.unipi.it

Received 12 July 2010; accepted 7 October 2010

\section{Introduction}

Involvement of the nervous system (central, peripheral, or autonomic) has been reported in varying 
frequencies ranging from $14-80 \%$ in patients with systemic lupus erythematosus (SLE). ${ }^{1-14}$ The ACR case definition for neuropsychiatric (NP) syndromes in SLE represents the most comprehensive attempt to classify clinical manifestations of neuropsychiatric SLE (NPSLE). However, it has been shown that some of the selected clinical symptoms have a low specificity (i.e. headache, mood disorders, mild cognitive dysfunctions). ${ }^{14-18}$

To date there has been little consensus on the role of laboratory, imaging and other diagnostic procedures in the diagnosis and follow-up of patients with NPSLE in routine clinical practice. Furthermore, many interventions are expensive, not readily available in daily practice and may carry considerable risks to patient health. ${ }^{8,9,19}$ Recently 'EULAR Recommendations for the Management of Systemic Lupus Erythematosus (SLE) with Neuropsychiatric Manifestations' have been developed, to guide in the assessment of patients with neuropsychiatric involvement. ${ }^{20}$ In this respect, the identification of patients less likely to have NPSLE versus those at major risk of having NPSLE by a simple screening questionnaire could simplify clinical practice and help in the decision to refer the patient for further assessment based on these recommendations.

The aim of the present project was the development of a questionnaire administered by physicians that could be useful in identifying patients with SLE and no obvious neuropsychiatric involvement who may be referred for further assessment with the suspicion of NPSLE.

\section{Patients and methods}

The development of the questionnaire followed three consecutive phases, namely: (i) items pool development and creation of a first draft questionnaire; (ii) weighting of the variables and items reduction; and (iii) testing on patients and development of the final questionnaire.

\section{Phase I: Items pool development and creation of a draft questionnaire}

Based on the ACR case definitions for NP syndromes in SLE, a list of manifestations was prepared. A subsequent literature search identified 41 questionnaires already in use for assessing patients with neurological and psychiatric manifestations similar to those reported in NPSLE (Table 1). ${ }^{21-59}$ A first draft questionnaire was then constructed including 119 items that were subdivided into
Central Nervous System (CNS), Peripheral Nervous System (PNS) and Psychiatric Manifestations (PsychM). Each possible answer received a score ranging from $-1-3$.

\section{Phase II: Items reduction and weighting of the variables}

The aim of this second phase was to simplify the first draft questionnaire by further reducing the number of questions in order to obtain a pilot version.

A Delphi analysis was carried out among the experts participating in the study. Experts were requested to judge whether each single question should be maintained or excluded. Questions excluded by more than $75 \%$ of the experts were removed from the list $(16 \%)$, questions excluded by less than $50 \%$ of the experts were retained $(8 \%)$; questions excluded by $50-75 \%$ of the experts were further discussed and re-evaluated (76\%).

The importance of each question was ranked on a Likert scale from 1-3: 1= irrelevant, non-important; 2 = somewhat relevant, somewhat important; $3=$ highly relevant, very important. The mean relevance scores for each item were calculated. Items with a mean rating $<1.5$ were removed; items with a mean rating $\geq 1.5$ and $<2$ had their scores halved; items with a mean rating $>2$ and $\leq 2.5$ had their scores unchanged; items with a mean rating $>2.5$ had their scores doubled.

A pilot version of the questionnaire including 62 questions was developed.

\section{Phase III: Testing on patients and} development of the final questionnaire

Each participating centre administered the pilot questionnaire to five SLE patients with active NPSLE, five SLE patients with previously diagnosed inactive NPSLE, five SLE patients with active disease and no history of NPSLE, and finally, five SLE patients with inactive disease and no history of NPSLE. The diagnosis of SLE was based on the 1997 revised ACR classification criteria. The diagnosis of NPSLE was made by the treating physician and represented the gold standard. In addition, global disease activity was also considered based on the ECLAM index: a score above 2 was considered as indicative of active disease, as previously published. ${ }^{19,20}$

The selection of the specific questions relevant to the screening of patients was performed by the receiver operating characteristic (ROC) curve analysis (Prism, Graph Pad Software Inc.) using the area under the curve (AUC) as selection criterion. The area ranges from 0.5 (no accuracy) to 1.0 
Table 1 List of the questionnaires used to assess neurological syndromes ${ }^{21-59}$

Acute confusional state

Acute Inflammatory Demyelinating

Polyradiculoneuropathy (Guillain-Barré Syndrome)

Anxiety disorder

Aseptic Meningitis

Autonomic disorder

Cerebrovascular Disease

Cognitive Dysfunction

Demyelinating Syndrome

Headache

Mononeuropathy (single/multiplex)

Mood Disorders

Movement Disorder (Chorea)

Myasthenia Gravis

Myelopathy

Neuropathy, Cranial

Plexopathy

Polyneuropathy

Psychosis

Seizures and Seizure Disorders
Confusion Assessment Method (CAM)

Delirium Observation Screening Scale

Delirium Rating Scale

No specific questionnaires found

Questions based on common clinical symptoms SCL-ANX4

Liebowitz Self-rated Disability Scale (LSRDS)

Clinician-Rated Disability Profile (DP)

Sheehan Disability Scale (SDS)

No specific questionnaires found

Questions based on common clinical symptoms

Autonomic Nervous System Response Inventory (ANSRI)

Daily Stress Inventory (DSI)

Somatic Response Survey

SCOPA-AUT

LOS Angeles Prehospital Stroke Screen (LAPSS)

The National Institutes of Health Stroke Scale (NIHSS)

Six-Item Screener test

Mini-Mental State Examination (MMSE)

Blessed Dementia Scale (BDS)

The 7-Minute Screen (7MS)

Questions based on common clinical symptoms

The MS Symptom and Impact Diary (MSSID)

The three-item ID Migraine

3-Question Headache Screen

Headache Disability Inventory (HDI)

Headache Impact Test (HIT)

Migraine Disability Assessment Score (MIDAS)

Migraine Specific Questionnaire (MSQ)

Migraine Therapy Assessment Questionnaire (MTAQ)

Headache Needs Assessment Survey (HANA)

Migraine Assessment of current Therapy (Migraine -ACT)

Pain Experience Instrument- Headache Version (CPEI-HA)

Questions based on common clinical symptoms

Neuropathic Pain Questionnaire (NPQ)

Neuropathic Pain Scale (NPS)

Leeds Assessment of Neuropathic Symptoms and Signs (LANSS)

Self-report Leeds Assessment of Neuropathic Symptoms and Signs (S-LANSS)

Depression screening with SIG and CAPS and with SALSA

Hamilton Rating Scale for Depression (HRSD)

Unified Huntington's Disease Rating Scale (UHDRS)

Myasthenia Gravis Questionnaire (MGQ)

Questions based on common clinical symptoms

Questions based on common clinical symptoms

Questions based on common clinical symptoms

Neuropathy Symptom Score

Questions based on common clinical symptoms

VA Seizures Frequency and Severity Scale (VA)

National Hospital Seizure Severity Scale (NHS3)

Occupational Hazard Scale

Liverpool Seizure Severity Scale Items

Hague Seizure Severity Scale (perfect accuracy). This method has the advantage of synthesizing information on the sensitivity and specificity for detecting improvement by an external criterion. Cut-off values to separate patients were established in order to maintain a high sensitivity $(>90 \%)$ and a specificity of at least $20 \%$.

\section{Results}

Out of the initial 119 questions, 65 were maintained after the first Delphi round. Based on the mean relevance scores for each item, three additional questions were excluded. Of the remaining 62, 21 had 
their scores halved, 35 had their scores unchanged and five had their scores doubled. These 62 questions constituted the pilot questionnaire.

In total, 139 SLE patients from 11 different European Centres (five in Italy, two in Spain, one in Lithuania, one in Poland, one in Portugal, one in The Netherlands) were enrolled in the study. Based on the NPSLE status, patients were distributed as follows: 58 patients with NPSLE, 29 active and 29 inactive at the time of investigation; 81 patients without history of NPSLE, 39 with active disease and 42 with inactive disease. Of NPSLE patients, $74 \%$ presented with CNS involvement (most frequently with vascular disease $38 \%$, epilepsy $21 \%$, and headache 9\%) 21\% PsychM, and 5\% PNS manifestations. PNS questions showed minimal predictive power for NPSLE, and therefore were excluded from the questionnaire.

In total, 27 questions from the CNS (12) and PsychM (15) groups were found to be relevant, while the remaining could be removed without significantly affecting the AUC of ROC curve (Figure 1). The final AUC was 0.69 (95\% CI $0.61-$ $0.78)$. The questionnaire is reported in Table 2.

Table 2 Questionnaire

\section{CENTRAL NERVOUS SYSTEM}

Headache

1. Do you frequently suffer from headaches (more than 1 episode every month?)

Yes

No

2. If YES

When did these headaches begin?

Before the onset of SLE

Before the onset of SLE, but worsening after After SLE onset

3. Does your headache improve after taking your daily dose of corticosteroids?

Never

Sometimes

Often

Always

Cerebrovascular Disease, TIA

4. Have you had (in the last 3 months), without warning, a sudden loss of the power or of sensation of an arm or of a leg?

Never

Yes, 1 episode

Yes, 2 episodes

Yes, more than 2 episodes

5. Have you been (in the last 3 months), without warning, suddenly unable to speak properly for less than 24 hours?

Never

Yes, 1 episode

Yes, 2 episodes

Yes, more than 2 episodes

Demyelinating syndrome

6. In the last 3 months have you had weakness or heaviness in your arms or legs?
Table 2 Continued

\section{CENTRAL NERVOUS SYSTEM}

Never

Yes, rarely

Yes, often

Yes, always

In the last 3 months have you had unsteadiness or loss of balance?

Never

Yes, rarely

Yes, often

Yes, always

8. In the last 3 months have you had alteration or loss of sensation (e.g. numbness, tingling, pins and needles) in some areas of your body?

Never

Yes, rarely

Yes, often

Yes, always

Never

Yes, rarely

Yes, often

Yes, always

Seizures

10. Do you suffer from seizures?

Yes

No

I don't know

Movement Disorder-Chorea

11. Have you had in the last 3 months abrupt, purposeless, nonrhythmic involuntary movements?

Never

Yes, rarely

Yes, often

Yes, always

Aseptic Meningitis

12. In the last 3 months have you had acute or gradual onset of headache with sensitivity to light, neck stiffness, confusion and fever? Never

Some time

Most of the time

All of the time

PSYCHIATRIC MANIFESTATIONS

Acute Confusional State and Cognitive dysfunctions

13. In the last 3 months, have you had difficulties in concentrating for a long time on the activities that you are doing?

Never

Sometimes

Most of times

Always

14. In the last 3 months, have you had difficulties in planning and managing your daily tasks or in organizing new activities?

Never

Sometimes

Most of times

Always recalling faces, routes, images?

Never

Sometimes

Most of time

Always

Mood Disorders

16. In the last 3 months, have you had sleep disturbances (insomnia with 2-4 am awakening)?

None of the time 
Table 2 Continued

\begin{tabular}{lc}
\hline CENTRAL NERVOUS SYSTEM & \\
\hline Some time & 0.5 \\
Most of the time & 1 \\
All of the time & 1.5 \\
17. In the last 3 months, have you had decreased interest in activities \\
(anhedonia)? & 0 \\
None of the time & 0.5 \\
Some time & 1 \\
Most of the time & 1.5 \\
All of the time & 0 \\
18. In the last 3 months, have you felt depressed? & 1 \\
Ever & 2 \\
Sometimes & 3 \\
Often & \\
Always & 0 \\
19. In the last 3 months, have you had suicidal thoughts? & 1 \\
None of the time & 2 \\
Some time & 3 \\
Most of the time &
\end{tabular}

Anxiety Disorder

20. In the last 3 months, have you felt more nervous and anxious than usual?

None of the time

Some time

Most of the time

All of the time

21. In the last 3 months, have you felt afraid for no reason at all?

None of the time

Some time

Most of the time

All of the time

Psychosis

22. In the last 3 months, have your relatives told you that you act in bizarre and inappropriate ways?

None of the time

Some time

Most of the time

All of the time

23. In the last 3 months, have you felt that you have more than one identity?

None of the time

Some time

Most of the time

All of the time

24. In the last 3 months, have you frequently repeated the same activities?

None of the time

Some time

Most of the time

All of the time

25. In the last 3 months, have you had impulsive unpredictable

behaviour?

None of the time

Some time

Most of the time

All of the time you have hallucinations?

None of the time

Some time

Most of the time

All of the time

27. In the last 3 months, have you thought of being persecuted?

None of the time

Some time

Most of the time

All of the time

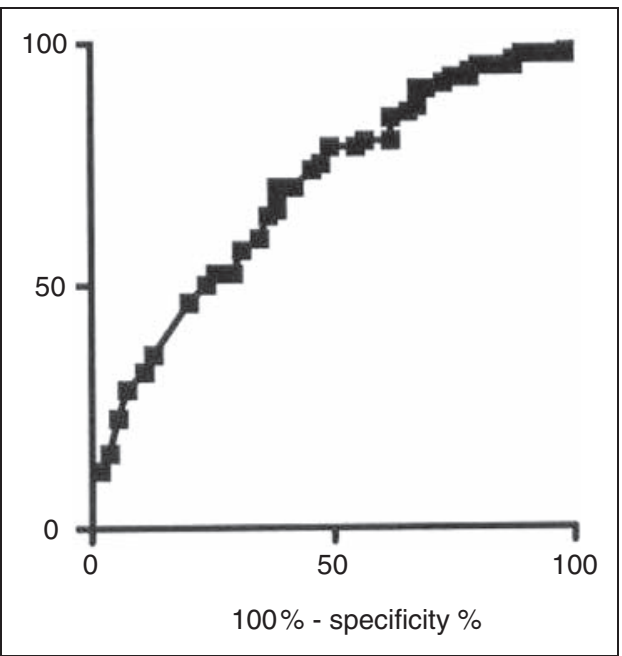

Figure 1 ROC Curve. A threshold of 17 points in the questionnaire score is able to distinguish a positive patient, with a sensitivity of $92.9 \%(95 \%$ CI $85.1-97.3 \%)$ and a specificity of $25.4 \%(95 \%$ CI $14.7-39.00 \%)$.

The best discriminating cut-off point corresponded to a score of 17 (i.e. a score higher than 17 to define a positive patient), for a sensitivity of $93 \%(95 \%$ CI $85.1-97.3 \%)$ and specificity of $25 \%$ (95\% CI 14.7-39.0\%). If CNS and PsychM were considered separately, the cut-off values identified to define positive patients were found to be 9 (sensitivity $90.6 \%$, specificity $23.3 \%$ ) and 10 (sensitivity $91.3 \%$, specificity $25.00 \%$ ) respectively.

\section{Discussion}

NP manifestations in SLE are very diverse and difficult to be evaluated; EULAR recommendations for monitoring NPSLE have been recently developed that offer a feasible guide in patient assessment. However, in routine clinical practice, patient screening for the presence of NPSLE is mainly based on a focused history, and patients are referred for further assessments according to the judgement of the treating physician. This leads to variability, as mild to moderate symptoms may be overlooked, depending on the physician's experience.

In the present study, we developed a simple questionnaire to screen patients with SLE for NP manifestations in routine clinical practice. This questionnaire should offer the treating physician a validated framework that supports the clinical examination, offering a core set of questions able to identify patients who should be referred for further assessment. 
The questionnaire includes 27 items covering CNS involvement and PsychM. Items referring to PNS manifestations turned out to be not relevant and were therefore excluded. A score above 17 was found to be suggestive of the presence of NP involvement and represents the cut-off value to refer patients for further assessment. This cut-off value offers a high sensitivity but a low specificity. However, since the aim of this questionnaire was to offer a first-level screening procedure, and by no means a tool for diagnosing such a complex condition as NPSLE, we have decided that the actual working point should be biased in favour of sensitivity.

The major objection raised by such an approach is the low specificity, leading to a high number of false-positive patients referred to the specialists. In fact, non-specific symptoms occurring in association (e.g. subjective complaint of cognitive impairment, low mood, headache), might score higher than more severe manifestations (e.g. seizures) when occurring alone. Although no corrections were made in the attribution of the scoring values, the final score was attributed on the basis of the panellists judgement on the relative importance of each question.

This questionnaire was developed to offer a simple screening tool to improve physician awareness of the presence of NP symptoms and should represent a guide in patient assessment in routine clinical practice. It is not intended to replace standard NP assessment of the patient with SLE. As in the case of any new neurological complaint, a positive screening result should be just a signal to the treating physician, who should evaluate the findings and draw the necessary conclusions.

At the opposite end of the spectrum, scores below the cut-off value that could be associated with a low suspicion of NPSLE should cautiously be judged by the treating physician.

Nonetheless, any screening questionnaire may help the physician in the assessment of patients, eventually reducing the unwanted variability. Such questionnaires need to be simple to administer and complete, and need to be acceptable to respondents. Short questionnaires minimize a patient's time and effort, and thus increase a patient's willingness to complete the questionnaire. To our knowledge, this is the first attempt to develop a simple questionnaire to screen patients for the presence of NPSLE to be used in clinical practice.

The absence of items referring to the presence of PNS involvement may be viewed as another limitation of this questionnaire. However, this reflects the relatively low prevalence of PNS involvement among patients with SLE.

Although the questionnaire was developed to be administered by the physician during assessment of the patient, in view of its simplicity it will also be tested as a patient-administered questionnaire.

In conclusion, this simple questionnaire may assist the treating physician in the screening of patients with SLE for the presence of non-overt NP involvement and provide a first-level evaluation before deciding on additional testing. This may avoid the delay in diagnosis and treatment.

\section{Acknowledgements}

We are thankful to Professor Ronald F Van Vollenhoven for his help in the preparation of the pilot questionnaire and comments on the final version of the manuscript.

\section{Funding}

This research received no specific grant from any funding agency in the public, commercial, or notfor-profit sectors.

\section{Conflict of interest}

The authors declare that they have no conflicts of interest.

\section{References}

1 Hanly JG, Su L, Farewell V, McCurdy G, Fougere L, Thompson K. Prospective study of neuropsychiatric events in systemic lupus erythematosus. J Rheumatol 2009; 36: 1449-1159.

2 Hanly JG, Urowitz MB, Su L, et al. Prospective analysis of neuropsychiatric events in an international disease inception cohort of SLE patients. Ann Rheum Dis 2010; 69: 529-535.

3 Hanly JG, Urowitz MB, Su L, et al. Short-term outcome of neuropsychiatric events in systemic lupus erythematosus upon enrollment into an international inception cohort study. Arthritis Rheum 2008; 59: 721-729.

4 Briani C, Lucchetta M, Ghirardello A, et al. Neurolupus is associated with anti-ribosomal $\mathrm{P}$ protein antibodies: An inception cohort study. J Autoimmun 2009; 32: 79-84.

5 Denburg SD, Stewart KE, Hart LE, Denburg JA. How "soft" are soft neurological signs? The relationship of subjective neuropsychiatric complaints to cognitive function in systemic lupus erythematosus. J Rheumatol 2003; 30: 1006-1010.

6 Petri M, Naqibuddin M, Carson KA, et al. Brain magnetic resonance imaging in newly diagnosed systemic lupus erythematosus. $J$ Rheumatol 2008; 35: 2348-2354.

7 Sanna G, Bertolaccini ML, Cuadrado MJ, et al. Neuropsychiatric manifestations in systemic lupus erythematosus: prevalence and 
association with anti-phospholipid antibodies. $J$ Rheumatol 2003; 30: 985-992.

8 Jennekens FG, Kater L. The central nervous system in systemic lupus erythematosus. Part 1. Clinical syndromes: a literature investigation. Rheumatology 2002; 41: 605-618.

9 Jennekens FG, Kater L. The central nervous system in systemic lupus erythematosus. Part 2. Pathogenetic mechanisms of clinical syndromes: a literature investigation. Rheumatology 2002; 41: 619-630.

10 Petri M, Naqibuddin M, Carson KA, et al. Cognitive function in a systemic lupus erythematosus inception cohort. J Rheumatol 2008; 35: 1776-1781.

11 Hanly JG. ACR classification criteria for systemic lupus erythematosus: limitations and revisions to neuropsychiatric variables. Lupus 2004; 13: 861-864.

12 Brey RL, Holliday SL, Saklad AR, et al. Neuropsychiatric syndromes in lupus: prevalence using standardized definitions. Neurology 2002; 58: 1214-1220.

13 Hanly JG, McCurdy G, Fougere L, Douglas JA, Thompson K. Neuropsychiatric events in systemic lupus erythematosus: attribution and clinical significance. $J$ Rheumatol 2004; 31: 2156-2162.

14 Ainiala H, Loukkola J, Peltola J, Korpela M, Hietaharju A. The prevalence of neuropsychiatric syndromes in systemic lupus erythematosus. Neurology 2001; 57: 496-500.

15 ACR ad hoc Committee on Neuropsychiatric Lupus Nomenclature. The American College of Rheumatology. Arthritis Rheum 1999; 42: 599-608.

16 Ainiala H, Hietaharju A, Loukkola $\mathrm{J}$, et al. Validity of the new American College of Rheumatology criteria for neuropsychiatric lupus syndromes: a population based evaluation. Arthritis Rheum (Arthritis Care Res) 2001; 45: 419-423.

17 Kozora E, Ellison MC, West S. Reliability and validity of the proposed American College of Rheumatology neuropsychological battery for systemic lupus erythematosus. Arthritis Rheum 2004; 51: 810-818.

18 Mosca M, Tani C, Aringer M, et al. EULAR Recommendations for monitoring systemic lupus erythematosus patients in clinical practice and in observational studies. Ann Rheum Dis 2010; 69: $1269-1274$.

19 Govoni M, Castellino G, Padovan M, Borrelli M, Trotta F. Recent advances and future perspective in neuroimaging in neuropsychiatric systemic lupus erythematosus. Lupus 2004; 13: 149-158.

20 Bertsias GK, Ioannidis $\mathrm{J}$, Aringer $\mathrm{M}$, et al. EULAR Recommendations for the management of systemic lupus erythematosus with neuropsychiatric manifestations: report of a task force of the EULAR standing committee for clinical affairs. Ann Rheum Dis 2010 Aug 19; doi: 10.1136/ard.2010.130476 [Epub ahead of print].

21 Gemert van LA, Schuurmans MJ. The Neecham Confusion Scale and the Delirium Observation Screening Scale: capacity to discriminate and ease of use in clinical practice. BMC Nurs 2007; 6: 3 .

22 Trzepacz PT. The Delirium Rating Scale. Its use in consultationliaison research. Psychosomatics 1999; 40: 193-204.

23 Gonzalez M, de Pablo J, Fuente E, et al. Instrument for detection of delirium in general hospitals: adaptation of the confusion assessment method. Psychosomatics 2004; 45: 426-431.

24 Søgaard HJ. Choosing screening instrument and cut-point on screening instruments. A comparison of methods. Scand J Public Health 2009; 37: 872-880.

25 Hambrick JP, Turk CL, Heimberg RG, Schneier FR, Liebowitz MR. Psychometric properties of disability measures among patients with social anxiety disorder. $J$ Anxiety Disord 2004; 18: 825-839.

26 Waters WF, Cohen RA, Bernard BA, Buco SM, Dreger RM. An Autonomic Nervous System Response Inventory (ANSRI): scaling, reliability, and cross-validation. J Behav Med 1984; 7: 315-341.

27 Dobkin PL, Pihl RO, Breault C. Validation of the Derogatis Stress Profile using laboratory and real world data. Psychother Psychosom 1991; 56: 185-196.

28 Visser M, Visser M, Marinus J, Stiggelbout AM, Van Hilten JJ. Assessment of autonomic dysfunction in Parkinson's disease: the SCOPA-AUT. Mov Disord 2004; 19: 1306-1312.
29 Waters WF, Rubman S, Hurry MJ. The prediction of somatic complaints using the Autonomic System Response Inventory (ANSRI) and the Daily Stress Inventory (DSI). J Psychosom Res 1993; 37: 117-126.

30 McCroskery JH, Reihman J. Development of the somatic response survey. Psychol Rep 1991; 68: 1097-1098.

31 Kidwell CS, Saver JL, Schubert GB, Eckstein M, Starkman S. Design and retrospective analysis of the Los Angeles Prehospital Stroke Screen (LAPSS). Prehosp Emerg Care 1998; 2: 267-273.

32 Hart CL, Hole DJ, Smith GD. The relation between question indicating transient ischaemic attack and stroke in 20 years of followup in men and women in the Renfrew/Paisley Study. $J$ Epidemiol Community Health 2001; 55: 653-656.

33 Goldstein LB, Simel DL. Is This Patient Having a Stroke? JAMA 2005; 19: 2391-2402.

34 Callahan CM, Unverzagt FW, Hui SL, Perkins AJ, Hendrie HC. Six-item screener to identify cognitive impairment among potential subjects for clinical research. Med Care 2002; 40: 771-781.

35 Madureira S, Guerreiro M, Ferro JM. Dementia and cognitive impairment three months after stroke. Eur J Neurol 2001; 80: 621-627.

36 Folstein MF, Folstein SE, McHugh PR. Mini-Mental State Examination (MMSE). A practical method for grading the cognitive state of patient for the clinician. J Psychiatr Res 1975; 12: 189-198.

37 Solomon PR, Hirschoff A, Kelly B, Relin M, Brush M, DeVeaux $\mathrm{RD}$. A 7-minute neurocognitive screening battery highly sensitive to Alzheimer's disease. Arch Neurol 1998; 55: 349-355.

38 Blessed G, Tomlinson BE, Roth $\mathrm{M}$. The association between quantitative measures of dementia and of senile change in the cerebral grey matter of elderly subjects. Br J Psychiatry 1968; 114: 749-811.

39 Beaumont JG. The MS Symptom and Impact Diary (MSSID): psychometric evaluation of a new instrument to measure the dayto-day impact of multiple sclerosis. J Neurol Neurosurg Psychiatry 2004; 75: 526-527.

40 Greenhalgh J, Ford H, Long AF, Hurst K. The MS Symptom and Impact Diary (MSSID): psychometric evaluation of a new instrument to measure the day-to-day impact of multiple sclerosis. J Neurol Neurosurg Psychiatry 2004; 75: 577-582.

41 Kosinski M, Bayliss MS, Bjorner JB, et al. A six-item short form survey for measuring headache impact: The HIT-6TM. Quality Life Res 2003; 12: 963-974.

42 Stewart WF, Lipton RB, Kolodner KB, Sawyer J, Lee C, Liberman JN. Validity of the Migraine Disability Assessment (MIDAS) score in comparison to a diary-based measure in a population sample of migraine suffers. Pain 2000; 88: 41-52.

43 Lipton RB, Kolodner K, Bigal ME, et al. Validity and reliability of the Migraine Treatment Optimization Questionnaire. Cephalalgia 2009; 29: 751-759.

44 Kilminster SG, Dowson AJ, Tepper SJ, Baos V, Baudet F, D'Amico D. Reliability, validity, and clinical utility of the Migraine-ACT questionnaire. Headache 2006; 46: 553-562.

45 Cramer JA, Silberstein SD, Winner P. Development and validation of the Headache Needs Assessment Survey. Headache 2001; 41: 402-409.

46 Dowson AJ, D'Amico D, Tepper SJ, Baos V, Baudet F, Kilminster S. Identifying patients who require a change in their current acute migraine treatment: the Migraine Assessment of Current Therapy (Migraine-ACT) questionnaire. Neurol Sci 2004; 25: s276-278.

47 Lipton RB, Dodick D, Sadovsky R, et al. A Self-administered screener for Migraine in Primary Care. The ID migraine validation study. Neurology 2003; 61: 375-382.

48 Cady RK, Borchert LD, Spalding W, Hart CC, Sheftell FD. Simple and efficient recognition of migraine with 3-question headache screen. Headache 2004; 44: 323-327.

49 Davis GC, Grassley JS. Measurement of the experience of living with primary recurrent headache. Pain Manage Nurs 2005; 6: $37-44$.

50 Bennett MI, Smith BH, Torrance N, Potter J. The S-LANSS score for identifying pain of predominantly neuropathic origin: Validation for use in clinical and postal research. J Pain 2005; 6: $149-158$. 
51 Bennett M. The LANSS Pain Scale: the Leeds assessment of neuropathic symptoms and signs. Pain 2001; 92: 147-157.

52 Krause SJ, Backonja MM. Development of a neuropathic pain questionnaire. J Pain 2003; 19: 306-314.

53 Conti L, Cassano GB. The impact of the Hamilton Rating Scale for Depression on the development of a center for clinical psychopharmacology research. Psychopharmacol Ser 1990; 9: 20-27.

54 Unified Huntington's Disease Rating Scale: reliability and consistency. Huntington Study Group. Mov Disord 1996; 11: 136-142.

55 Padua L, Galassi G, Ariatti A, et al. Myasthenia gravis selfadministered questionnaire: development of regional domains. Neurol Sci 2005; 25: 331-336.
56 Cramer JA. A clinimetric approach to assessing quality of life in epilepsy. Epilepsia 1993; 34(Suppl 4): S8-13.

57 O'Donoghue MF, Duncan JS, Sander JW. The National Hospital Seizure Severity Scale: a further development of the Chalfont Seizure Severity Scale. Epilepsia 1996; 37: 563-571.

58 Cramer JA, French J. Quantitative assessment of seizure severity for clinical trials: a review of approaches to seizure components. Epilepsia 2001; 4: 119-129.

59 Cramer JA. Assessing the severity of seizures and epilepsy: which scales are valid? Curr Opin Neurol 2001; 14: 225-229. 